\title{
Autobiografías de enfermeras: la profesionalización de enfermería durante las guerras*
}

\author{
Autobiography of nursing: \\ nursing professionalization during the war \\ Autobiografias de enfermeiros:
a profissionalização da enfermagem na guerra
}

Ana Choperena Armendáriz ${ }^{1}$

${ }^{1}$ Licenciada en Humanidades y Diplomada en Enfermería. Máster en Ciencias de la Enfermería. Profesora Colaboradora Historia de la Enfermería. Facultad de enfermería Universidad de Navarra.

Cómo citar este artículo en edición digital: Choperena Armendáriz, A. (2012) Autobiografías de enfermeras: la profesionalización de enfermería durante las guerras.

16, 34. Disponible en: <http://dx.doi.org/10.7184/cuid.2012.34.06>

Correspondencia: Ana Choperena Armendáriz C/ Irunlarrea, 1, 31008. Pamplona (Navarra). Mail: achoperena@unav.es Recibido 03/02/2012/ Aceptado: 08/06/2012

* III Simposio iberoamericano de historia de la enfermería, XII congreso nacional, VII internacional de historia de la enfermería y VII jornadas internacionales de cultura de los cuidados. Alicante 2011.

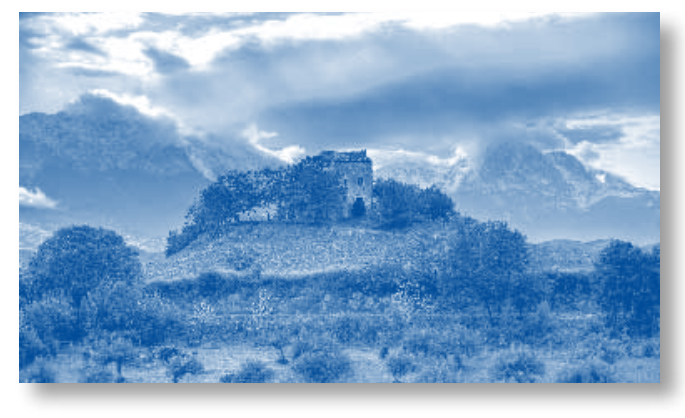

ABSTRACT

This paper proposes an historical analysis of a group of nurses whose autobiographies of dramatic experiences in the exercise of his profession during the American Civil War helped to shape the nursing profession.

The autobiographies are an important component of self-perception that not only becomes a way of conveying a reality from the subjective point of view and personal,

but also helps shape a collective identity.

Thus, the historical analysis of the experiences of nurses can lead to understanding of the nature of a young profession that came in the nineteenth century and that has taken shape over the twentieth century, reaching a clearly defined status and self-discipline with its development and specific practice.

We have chosen the context of the American Civil War because of its impact in the professional development of nursing. It is well known that the experience of the dramatic events excites reflection after a while, and often results in retrospective autobiographical accounts that reflect the referential aspect of events.

Keywords: Nursing, Autobiography, History, Self-Knowledge, American Civil War.

\section{RESUMO}

Este artigo propõe uma análise histórica de um grupo de enfermeiros cujas autobiografias de experiências dramáticas no exercício de sua profissão durante a Guerra Civil americana ajudou a moldar a profissão de enfermagem.

As autobiografias são um componente importante de auto-percepção de que não só se torna uma maneira de transmitir uma rea- 
lidade do ponto de vista subjetivo e pessoal, mas também ajuda a moldar uma identidade coletiva.

Assim, a análise histórica das experiências de enfermeiros pode levar à compreensão da natureza de uma profissão jovens que vieram no século XIX e que tomou forma ao longo do século XX, atingindo um estatuto claramente definido e auto-disciplina com seu desenvolvimento e prática específica.

Nós escolhemos o contexto da Guerra Civil Americana por causa de seu impacto no desenvolvimento profissional de enfermagem. É bem sabido que a experiência dos acontecimentos dramáticos excita reflexão depois de um tempo, e muitas vezes resulta em relatos autobiográficos retrospectiva que refletem o aspecto referencial de eventos.

Palavras-chave: Enfermagem, Autobiography, História, Auto-Conhecimento, Guerra Civil americana.

\section{RESUMEN}

Este trabajo propone el análisis histórico de un grupo de autobiografías de enfermeras cuyas dramáticas experiencias en el ejercicio de su profesión durante la Guerra Civil Americana ayudaron a configurar la profesión de enfermería.

Las autobiografías tienen un importante componente de auto-percepción que no sólo les convierte en un modo de transmitir una realidad desde el punto de vista más subjetivo y personal, sino que también ayuda a configurar la identidad de un colectivo.

De esta manera, el análisis histórico de las experiencias de enfermeras puede conducir al conocimiento de la naturaleza de una joven profesión que nació en el siglo XIX y que se ha ido configurando a lo largo del XX, alcan- zando un estatus perfectamente definido y una disciplina propia, con su desarrollo y práctica específica.

Se ha escogido el contexto de la Guerra Civil Americana por su repercusión en cuanto al desarrollo profesional de la enfermería. Es bien sabido que la experiencia de los acontecimientos dramáticos excita la reflexión personal al cabo de un tiempo, y en muchas ocasiones da lugar a relatos autobiográficos retrospectivos que reflejan el aspecto referencial de los acontecimientos.

Palabras clave: Enfermería, Autobiografía, Historia, Autoconocimiento, Guerra Civil Americana.

\section{INTRODUCCIÓN}

El género autobiográfico tiene un importante componente de autoconocimiento, que no sólo lo convierte en un modo de transmitir una realidad desde el punto de vista más subjetivo y personal, sino que además, ayuda a configurar la identidad de un colectivo, que en este caso afecta a la profesión de enfermería.

El conocimiento del pasado de la enfermería puede convertirse en un medio para hacer frente al futuro gracias a la proyección de experiencias ya acontecidas y puede mostrar que lo que hoy existe de una manera, no fue siempre así.

La tradición autobiográfica de enfermeras no es copiosa. Este aspecto, motivado por la influencia de una sociedad en la que las mujeres no se sentían capaces o incluso libres para hablar de sí mismas, ha llevado a que los relatos autobiográficos de enfermeras durante la Guerra civil Americana no abunden. Es indudable que dicha escasez acrecienta el interés de dichos relatos, tanto desde el punto de vista específico que se propone ahora (conocimiento) 
como desde el punto de vista, estrictamente, de la crítica literaria en torno al género autobiográfico.

En el estudio que aquí se propone, se ha escogido el contexto de la Guerra Civil Americana por su repercusión en el desarrollo de la profesión de enfermería. Además, es bien sabido que la experiencia de los acontecimientos dramáticos excita la reflexión personal al cabo de un tiempo y, en muchas ocasiones, da lugar a relatos autobiográficos retrospectivos que refuerzan la conciencia colectiva de una profesión.

Con todo esto, se propone el estudio de autobiografías de enfermeras que trabajaron durante la Guerra Civil Americana como fuente de conocimiento de los inicios profesionales de la enfermería.

\section{ESTADO DE LA CUESTIÓN}

Los estudios de formación y consolidación de una identidad profesional colectiva, utilizando las autobiografías como fuente, se han llevado a cabo en distintos campos. Destacan, particularmente, los llevados a cabo para la profesión de los historiadores. El libro de Jeremy Popkin, History, Historians \& Autobiography constituye un buen modelo para el análisis de la configuración de la identidad profesional de las enfermeras que se propone.

En cuanto a los estudios que analizan escritos personales de enfermeras durante la Guerra Civil americana, cabe destacar los trabajos de Jane E. Schultz, Sister Mary Denis Maher, Mary Ann Cordeau, Cornelia Sexauer y Mercedes Graf. Hasta el momento, no se ha encontrado ningún otro trabajo que, de manera específica, trate los géneros autobiográficos en esta guerra y su repercusión para la enfermería. Ante este panorama, la utilidad del estudio que se propone queda reforzada.

\section{AUTOBIOGRAFÍA, HISTORIA Y AUTO- CONOCIMIENTO}

Resulta complicado establecer una definición universal de autobiografía. El género autobiográfico surgió, en un primer momento, como una rama de la biografía. Dilthey (1914) fue el primero que supo ver la importancia de la autobiografía como herramienta de percepción histórica (Eakin, 1992:.182) y propuso la aproximación a la historia a través de la autobiografía. Así, se puede hacer una verdadera historia intelectual a través de textos autobiográficos (Aurell, 2005).

El conocimiento de la historia de la enfermería sirve para comprender tanto el presente de la profesión como el futuro, gracias a la proyección del pasado. De hecho, la experiencia directa de los acontecimientos no es, necesariamente, la mejor senda hacia su comprensión puesto que el campo visual puede que no se extienda mucho más allá de los sentidos inmediatos (Gaddis, 2004). La historia de la enfermería difícilmente puede transmitirse y fijarse de otro modo que no sea a través de la escritura, del texto histórico.

Las circunstancias de las vidas de las enfermeras son un testimonio elocuente del rastro histórico que ellas mismas han dejado, al tiempo que condicionan el modo actual de percibir el pasado. Las enfermeras que relatan sus experiencias en la guerra, toman distancia de los acontecimientos, se elevan y trascienden el ámbito de lo inmediato. Así, además de sujetos que experimentan una serie de vivencias, se convierten en testigos privilegiados de la historia. Por todo esto, más allá de una mera reproducción de la experiencia:

- between the parts we see a connection which neither is, nor is it intended to be, the simple likeness of the course of a life of so many years, but which, because understan- 


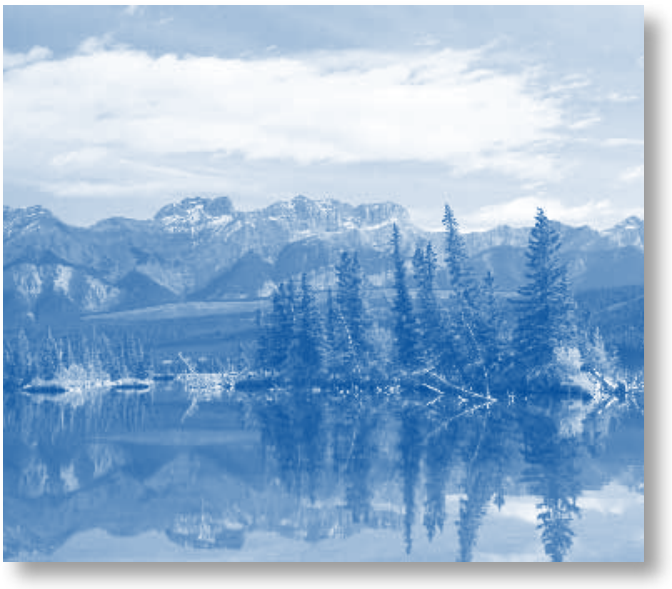

ding is involved, expresses what the individual knows about the continuity of his life (Dilthey, 1962: 85-86).

Por tanto, la autobiografía posibilita la comprensión de las experiencias vitales de manera enlazada, como un todo con sentido global.

Al género autobiográfico se le atribuye la capacidad de provocar y posibilitar el autoconocimiento, no sólo del autor, sino también del lector. Según Olney, as readers we go to history, as to philosophy, to autobiography and poetry, to learn more not about other people and the past but about ourselves and the present $(\mathrm{Ol}-$ ney, 1972:36-37). Popkin admite que, cada vez que lee memorias de historiadores, éstas le llevan a reflexionar sobre las características, no sólo de su profesión, sino de su propia vida. La lectura de relatos autobiográficos de enfermeras puede conducir al conocimiento de las raíces de la profesión y a la profundización en el conocimiento propio.

El autor de un relato autobiográfico puede describir el pasado desde dentro y desbordar motivos y reacciones que un observador externo nunca hubiera percibido (Popkin, 2005:6). Un relato histórico puede convertirse en un texto árido y estéril que se limite a la narración de unos hechos carentes de sentido por sí mis- mos. En cambio, las enfermeras que trabajaron en la Guerra Civil Americana pueden transmitirnos sentimientos y emociones, en definitiva, significados que conducen al conocimiento.

\section{FUENTES PRIMARIAS}

La experiencia de la Guerra Civil Americana supuso para el mundo de la enfermería no sólo los albores de una profesión que se venía ya forjando gracias a la figura de Florence Nightingale sino también la toma de conciencia de la importancia de un conjunto de mujeres que tuvieron en sus manos los cuidados de miles de soldados y heridos. A través de las fuentes primarias que se conservan de algunas de aquellas protagonistas se puede alcanzar a conocer sus experiencias vitales. Dichas fuentes sirven como modelo de identificación y de aprendizaje. La lectura de sus relatos autobiográficos, posibilita la toma de conciencia de la identidad profesional de la enfermería y, de esta manera, el conocimiento del pasado cumple con su función.

Las guerras forman parte del pasado y se recurre a ellas para el conocimiento, en este caso, de la profesión de enfermería. La guerra funciona como un símbolo familiar de experiencia colectiva y puede servir de conexión, a través de la autobiografía, entre la referencia biográfica y la referencia histórica (Eakin, 1992:179).

Tanto los acontecimientos públicos como los privados forman parte por igual del entramado de la vida y el punto a partir del cual los consideramos separados es ficticio. Esto lleva a que, tanto los aspectos históricos como los puramente biográficos de los relatos que se proponen resulten útiles a la hora de configurar la historia de la profesión. De hecho, los historiadores han recurrido, en numerosas ocasiones, al análisis histórico de narraciones autobio- 
gráficas en torno al fenómeno de la guerra y han reflejado cómo esa experiencia contribuyó en el desarrollo, no sólo de su profesión, sino de sus propias vidas. Incluso, las experiencias de guerra son tomadas, en ocasiones, como acontecimientos más determinantes que la propia infancia. Con todas estas aportaciones, los escritos autobiográficos de guerra contribuyen a la comprensión de las experiencias humanas.

Así pues, el contexto de la Guerra Civil Americana se convierte en el medio adecuado para alcanzar el conocimiento de los inicios de la profesión. Las circunstancias de las vidas de las enfermeras que desempeñaron los cuidados de los heridos y enfermos son un testimonio elocuente del rastro histórico y profesional que ellas mismas fueron dejando, al tiempo que condicionan la percepción del pasado y del presente y la proyección del futuro.

\section{CONCLUSIONES}

El género autobiográfico puede emplearse como herramienta para la configuración de la historia profesional de la enfermería. El componente de autoconocimiento presente en las autobiografías puede colaborar en el fortalecimiento de la identidad profesional de la enfermería actual.

El análisis histórico de los relatos autobiográficos de enfermeras puede aportar matices que enriquezcan la comprensión general de la disciplina.

Las circunstancias de las vidas de las enfermeras que desempeñaron los cuidados de los heridos y enfermos durante la Guerra Civil Americana son un testimonio elocuente del rastro histórico y profesional que ellas mismas fueron dejando, al tiempo que condicionan la percepción del pasado y del presente y la proyección del futuro.

\section{BIBLIOGRAFÍA}

- Aurell, J.(2005) La escritura de la memoria. Universitat de Valencia, Valencia.

- Cordeau, M. A. (2009) A Method for Historicizing Lived Experience. Advances in Nursing Science, 32 (1), 75-90.

- Dilthey, W. (1962) Pattern and Meaning in History: Thoughts on History and Society. Ed. H. P. Rickman. Harper and Row, New York.

- Eakin, P.aul J.(1992) Touching the World. Megazul-Endymion, Madrid.

Graf, M. (2010). On the Field of Mercy. Women Medical Volunteers from the Civil War to the First World War. Humanity Books, New York.

- Gaddis, J.L. (2004) El Paisaje de la Memoria. Anagrama, Barcelona.

- Maher, Sister M.D. (1999) To bind Up the Wounds: Catholic Sister Nurses in the U.S. Civil War. Louisiana State University Press, Lousiana

- Olney, J. (1972) Metaphors of Self: The Meaning of Autobiography. Princeton University Press, . Princeton, N.J.

- Popkin, J.D. (2005) History, Historians \& Autobiography. University of Chicago Press, Chicago.

- Schultz, J.E. (2004) Women at the Front: Hospital Workers in Civil War America. The University of North Carolina Press, North Carolina.

- Sexauer, C. (2002). Untold Stories: Women in the Civil War. CRM, (4), 30-32. 